\title{
Broadband Backhaul Communication for Stratospheric Platforms: The Stratospheric Optical Payload Experiment (STROPEX)
}

\author{
Joachim Horwath ${ }^{1}$, Markus Knapek, Bernhard Epple, Martin Brechtelsbauer \\ German Aerospace Centre (DLR), Institute of Communications and Navigation, Weßling, Germany. \\ Brandon Wilkerson \\ U.S. Air Force Exchange Engineer at the German Aerospace Centre
}

\begin{abstract}
A high bitrate optical downlink was performed by the stratospheric optical payload experiment (STROPEX), a part of the EU CAPANINA project. The STROPEX objectives were to design and build the necessary hardware to demonstrate an optical backhaul downlink from a stratospheric platform to the ground and to carry out channel measurements on the link. A successful measurement campaign at ESRANGE near Kiruna, Sweden achieved all of these objectives. The transportable optical ground station received an almost error free $1.25 \mathrm{Gbit} / \mathrm{s}$ data signal from the payload over a distance of $64.3 \mathrm{~km}$ with a bit error rate of better than $10^{-9}$. This paper gives an overview of the stratospheric optical payload experiment, focusing on the airborne free-space experimental laser terminal (FELT). Additionally, the successful measurement campaign is described and the operation of the experiment is outlined.
\end{abstract}

Keywords: Optical Inter Platform Link, High Altitude Platform, CAPANINA, STROPEX, stratospheric environmental conditions

\section{INTRODUCTION}

Communication from High Altitude Platforms (HAPs) has the potential to fill a gap between terrestrial and satellite communication. Aerodynamic aircraft or aerostatic platforms hovering quasi-geostationary at an altitude of 18 to $25 \mathrm{~km}$ could combine the advantages of both established infrastructures. Some advantages include short deployment time and easy equipment upgrade, flexible capacity increase through spot-beam re-sizing and additional platforms, ability of substantial indoor coverage, and a geographical coverage of hundreds of kilometers. The European CAPANINA project is developing communications technologies for use with aerial platforms with the aim of delivering "broadband for all." The emphasis is on hard to reach users and those disadvantaged by geography [1]. Services like fixed broadband wireless access up to $120 \mathrm{Mbit} / \mathrm{s}$ to an end user or to users traveling at high speeds (e.g. in a train) require a broadband backhaul network [2].

Free-space optical (FSO) communication technology has a major potential to complement microwave technology for the backhaul traffic. High attenuation due to clouds is not a limiting factor for inter-platform and platform to satellite links, the main application of HAP-FSO. Due to a maximum cloud ceiling of $13 \mathrm{~km}$ for mid-latitude locations, inter platform link distances of up to $900 \mathrm{~km}$ are possible with $100 \%$ availability [3]. For the optical downlink, large scale cloud cover diversity techniques can be used to supplement the microwave downlink and save platform power during FSO terminal operation.

In order to design reliable optical terminals, stratospheric tests are necessary. The CAPANINA Stratospheric Optical Payload Experiment (STROPEX) was one step in this direction by gaining system design and operations experience and by gathering atmospheric index-of-refraction turbulence data. The experiment was focused on experimental verification of the acquisition, pointing, and tracking systems; measurement of atmospheric impacts; and successful verification of a broadband downlink from a stratospheric test-bed (balloon/aerodynamic HAP aircraft).

${ }^{1}$ Contact Author: Joachim Horwath is with the German Aerospace Center, Institute for Communications and Navigation, Oberpfaffenhofen, 82234 Wessling, Germany (phone: ++49-8153-281832; fax: ++49-8153-282844; e-mail: joachim.horwath@ dlr.de). Manuscript received July 21, 2006.

Copyright 2006 Society of Photo-Optical Instrumentation Engineers. This paper was published in Proceedings of the SPIE Vol. 6304 and is made available as an electronic reprint with permission of SPIE. One print or electronic copy may be made for personal use only. Systematic or multiple reproduction, distribution to multiple locations via electronic or other means, duplication of any material in this paper for a fee or for commercial purposes, or modification of the content of the paper are prohibited. 


\section{SYSTEM DESCRIPTION}

\subsection{System Concept}

The overall system concept for STROPEX was to mount a free-space optical terminal (FELT) on a stratospheric balloon that would fly to an altitude of approximately $22 \mathrm{~km}$. A downlink would then take place between the FELT and a Transportable Optical Ground Station (TOGS) as depicted in Fig. 1.

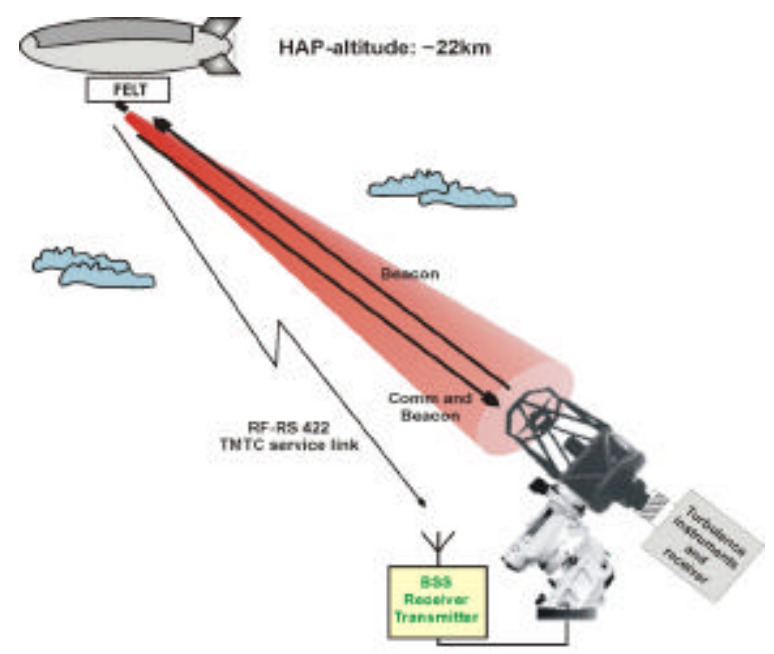

Optical Ground Station

Fig. 1. STROPEX Scenario

Fig. 2 shows a block diagram highlighting the components included on the FELT and the TOGS. The optical communication transmission occurred only on the downlink (i.e. from the FELT to the TOGS) whereas a RF-RS422 service link from the balloon service system (BSS) was used to communicate with and operate the FELT.

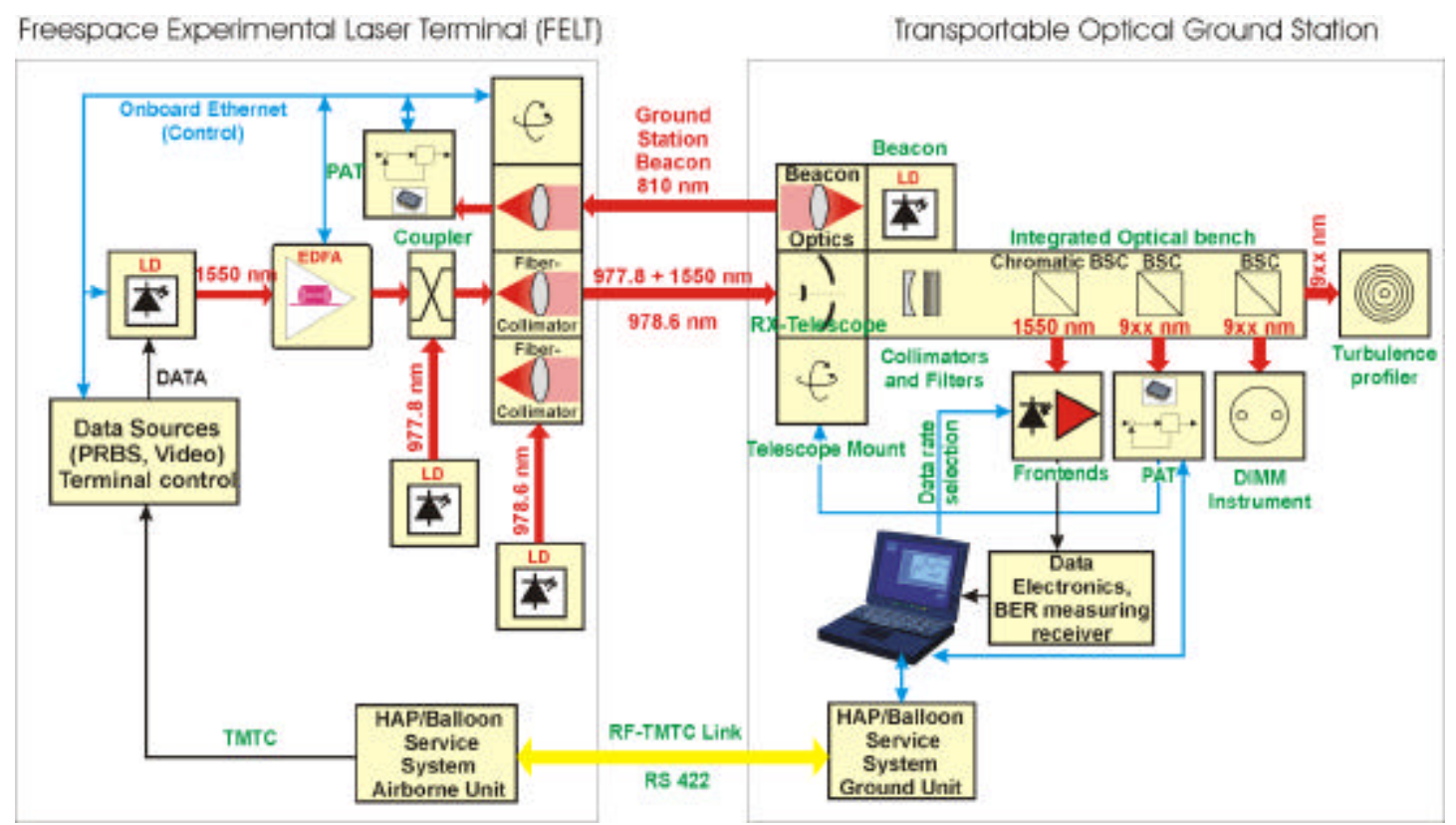

Fig. 2. Block Diagrams of the FELT and TOGS

Based on various factors such as component availability and atmospheric attenuation [3], three different wavelengths were chosen for the lasers. The TOGS used beacon lasers at $810 \mathrm{~nm}$ to illuminate the FELT. The FELT had two beacon 
lasers and a communication laser at $1550 \mathrm{~nm}$. At $1550 \mathrm{~nm}$, the atmospheric absorption is negligible in clear air conditions which make it a favorable wavelength for FSO applications [3].

Intensity modulation (IM, on/off-keying) with direct detection (DD), common in terrestrial fiber-optical transmission, was the chosen transmission scheme. An IM/DD scheme is advantageous because there are a range of available components with proven reliability e.g. laser diodes, fiber amplifiers, detectors and receiver electronics. According to theory, 10 incoming photons per bit (mean) are sufficient for an uncoded Bit-Error-Rate (BER) of 10 $0^{-9}$. However, in practical systems using standard APD-detectors (avalanche photodiode), the receiver sensitivity is usually not better than 500 Photons per bit due to thermal receiver noise and other degrading electronic effects. The actual sensitivity of the frontend developed for STROPEX was measured at 168 Photons per bit at a data transmission rate of $1.25 \mathrm{Gbit} / \mathrm{s}$ and a bit error rate of $3 \times 10^{-7}$.

\subsection{Free Space Optical Terminal (FELT)}

The Free-space Experimental Laser Terminal (FELT) was an optical transmission terminal developed mainly for the CAPANINA stratospheric experiments. The following were the primary design constraints for the FELT:

- Stratospheric environmental conditions (temperatures down to $-70^{\circ} \mathrm{C}$ and near vacuum conditions)

- High possible rotation speeds of the stratospheric carrier (9 rotations per minute).

- $\quad$ Lightweight and streamlined design for a future trial with an aerodynamic HAP

- Autonomous and robust acquisition capability in the presence of strong background light

In under a year, the FELT was designed, built, tested, and flown. This included numerous subsystem prototype developments, thermal vacuum chamber tests, and the development of several thousand lines of program code that was implemented into the two onboard computers of the terminal. Before flight, the overall system was tested for one month at a $28 \mathrm{~km}$ test range in the Bavarian Alps.
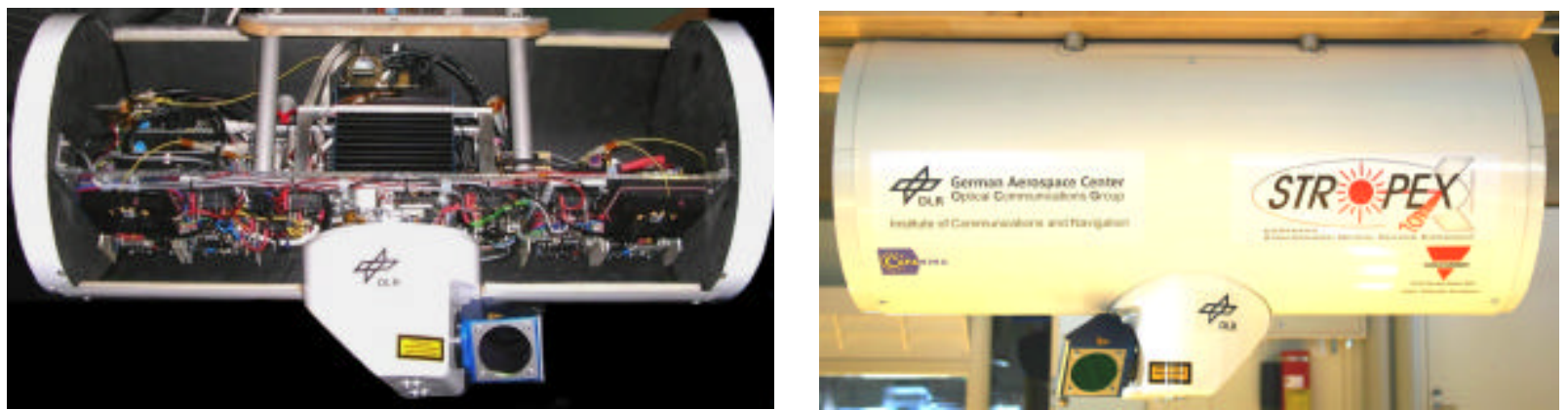

Fig. 3. Assembly photo of FELT with opened carbon fiber housing (left) and the ready to fly terminal (right) before payload integration.

The final FELT design is shown in Fig. 3. The structural subsystem consisted of an aluminum base plate which was also used for passive thermal control. The objective design weight was $25 \mathrm{~kg}$ and the final terminal weight was $17.54 \mathrm{~kg}$ thanks in part to the payload housing developed by project partner Carlo Gavazzi Space SpA. The housing consisted of a carbon fiber sandwich structure that reduced weight and maximized thermal insulation properties. Additionally, the housing was designed to easily interface with an aerodynamic HAP (i.e. a stratospheric airplane). The IMT 2000 payload, developed by project partner, NICT, served as a design prototype [4].

Fig. 4 shows the bottom side of the mounting plate, where the electrical power subsystem along with the beacon laser diodes and their supporting electronics (diode drivers and thermal control) were mounted. For redundancy, the FELT used two independent co-aligned beacon systems at $978.6 \mathrm{~nm}$ and $977.8 \mathrm{~nm}$, each with $200 \mathrm{~mW}$ output power. One of the beacons was coupled with the $1550 \mathrm{~nm}$ signal light and used the same transmission optics. The pointing assembly consisted of a 2-axis optical periscope along with the supporting encoders, DC motors, and motor drivers. The pointing resolution was $8.7 \mu \mathrm{rad}$ with a maximum rotation speed of $240 \% \mathrm{~s}$ for the main axis and $120 \% \mathrm{~s}$ for the elevation axis. The 
acceleration was better than $250 \% \mathrm{~s}^{2}$. The design driver for the dynamic properties of the pointing assembly was the possible high spin rate of the stratospheric balloon ( 9 rotations per minute). Five DC-DC converters were also mounted on the bottom side of the plate. The balloon power system provided $28 \mathrm{~V}$ and $24 \mathrm{~V}$ power sources to the FELT which were converted to $\pm 3.3 \mathrm{~V}, 5 \mathrm{~V}$ and $12 \mathrm{~V}$.

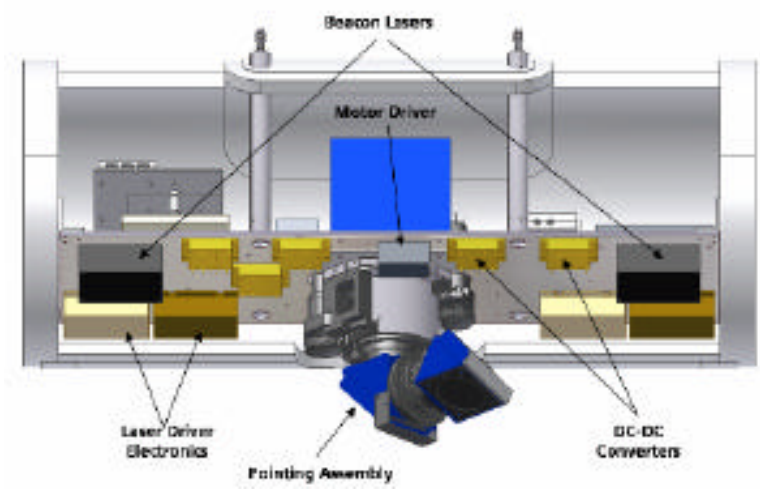

Fig. 4. CAD sketch of FELT - Bottom side.

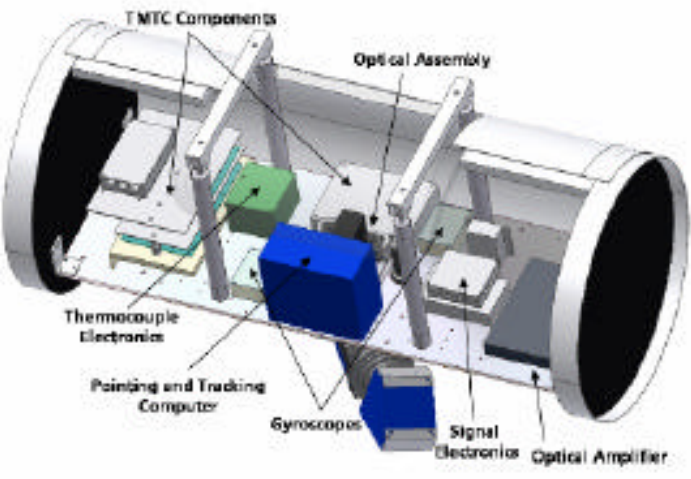

Fig. 5. CAD sketch of FELT - Top side.

The components mounted on top of the plate can be seen in Fig. 5. An onboard Telemetry and Tele-Command (TMTC) subsystem enabled control of the terminal via RS422 connection and included the monitoring of 16 temperature probes. The signal electronics generated a pseudo random binary sequence (PRBS) with a sequence length of $10^{23}-1$ and an adjustable data rate of up to $1.25 \mathrm{Gbit} / \mathrm{s}$. This data source drove a laser diode module with $1 \mathrm{~mW}$ output power which was optically amplified to a transmission power of $100 \mathrm{~mW}$. The beam was routed into the pointing assembly via the optical assembly, which consisted of the laser collimators, optical components, and the fast CMOS tracking camera with a $4^{\circ}$ FOV in the acquisition mode [12]. The pointing assembly was controlled by the FELT Pointing Acquisition and Tracking (PAT) computer. The PAT computer took inputs from on-board gyroscopes, the tracking camera, and the periscope encoders and controlled the motion of the pointing assembly. The FELT characteristics are summarized in Table 1.

Table 1: FELT Characteristics

\begin{tabular}{|l|l|}
\hline Mass & $17.5 \mathrm{~kg}$ \\
\hline Power Consumption & $<75 \mathrm{Watts}$ \\
\hline Communication Rate & Up to $1.25 \mathrm{Gbit} /$ second \\
\hline Laser wavelengths & $9 \mathrm{xx} \mathrm{nm}$ (beacons) \\
& $1550 \mathrm{~nm}$ (communication) \\
\hline Power busses & $\pm 3.3 \mathrm{~V}, 5 \mathrm{~V}$ and $12 \mathrm{~V}$ \\
\hline
\end{tabular}

\subsection{Optical Assembly}

The FELT optical system consisted of the tracking camera for the incoming ground station beacon and the fiber coupled transmission system. A sketch can be seen in the left part of Fig. 6. The initial design featured a chromatic beam splitter which would have had the advantage of both the transmission system and the tracking system being able to use the full aperture size. However, due to the fact that the commissioned chromatic beam splitter parts could not be delivered in time, the optical assembly was changed to a relatively simple layout. The tracking system aperture was reduced and coaligned transmission systems were used. A design challenge was the precise co-alignment of all optical axes, which had to remain aligned within a temperature range of $120^{\circ} \mathrm{C}\left(-70\right.$ to $\left.+50^{\circ} \mathrm{C}\right)$. A tempered aluminum alloy was used to mill the optical assembly part. Numerous tests with different glues for the fiber collimators were necessary to achieve the targeted specifications. One of the prototype systems can be seen in Fig. 6 (right) during the assembly process. 
The pointing assembly concept based on the two-axis periscope is very flexible. Besides the reduction of moved mass, various optical assemblies can be attached to the pointing assembly. Additionally, modification to a transmit/receive terminal (instead of just a transmit terminal) can be done just by changing the optical assembly.

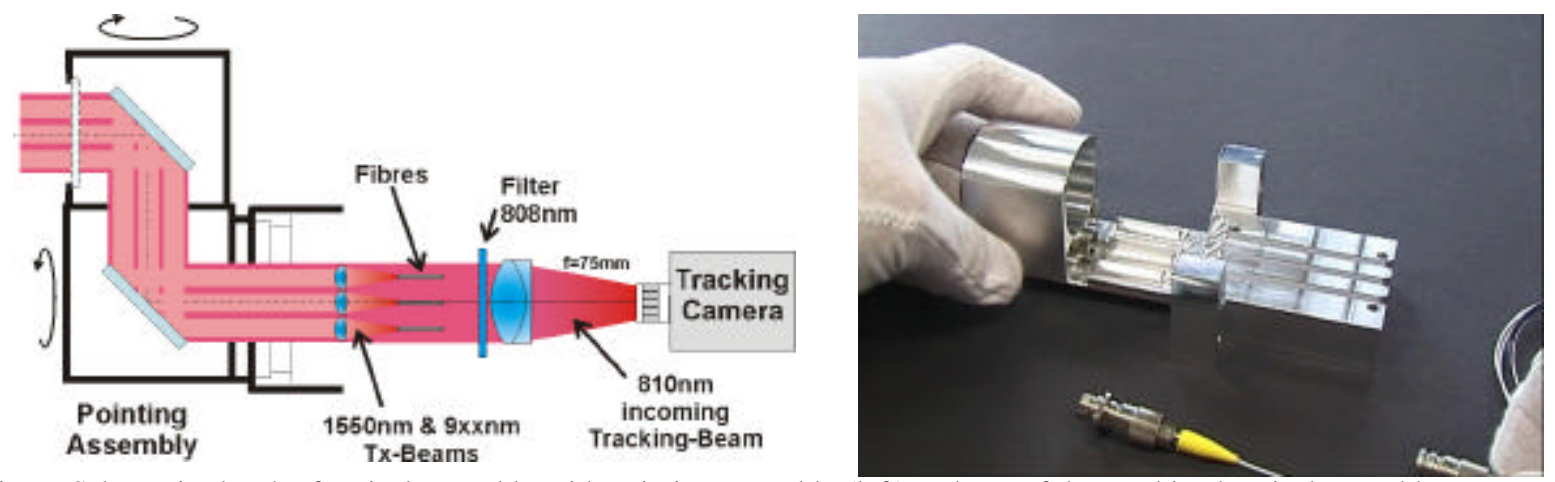

Fig. 6: Schematic sketch of optical assembly with pointing assembly (left) and one of the machined optical assembly prototypes during the assembly of fibres (right)

\subsection{Data electronics}

The FELT Communication system was comprised of the data sources, data switches, and the laser transmitter. Fig. 7 shows an overview block diagram of the system including the optical transmitter modules and the EDFA from the optical subsystem. The control circuits (Ethernet I/O) were connected to the onboard Ethernet. The maximum data rate of 1.244 GBit/s was chosen based on availability of the commercial laser-transmitter module. A special kind of module which could cover the entire range between the maximum data rate and the low rate of $10 \mathrm{Mbps}$ was required. This demand could not be fulfilled by commercial $2.5 \mathrm{Gbit} / \mathrm{s}$ modules. The capability to transmit at low bit rates was included to allow for optical communications with decreased signal to noise ratios, which could have occurred on the channel (e.g. increased attenuation due to clouds).
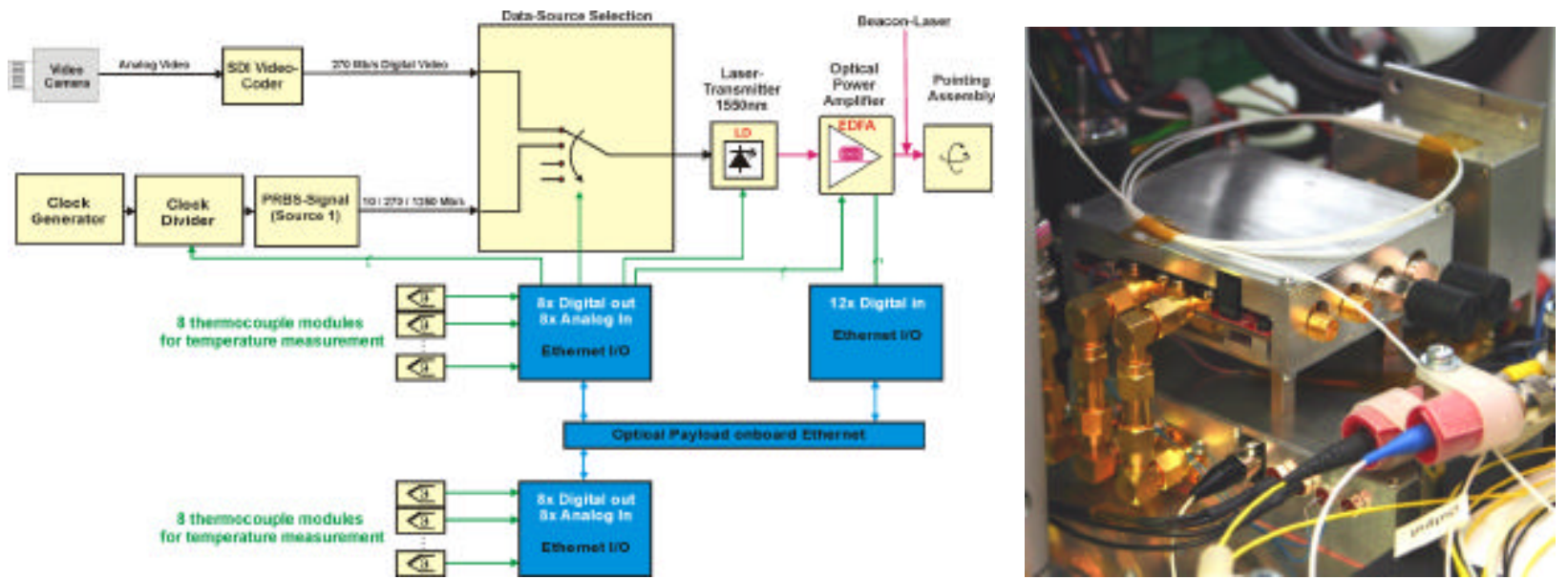

Fig. 7: Block diagram of FELT Data electronics (left) and photo of the system during integration into the FELT (right). The upper housing with the wound-up fiber on top is the pseudo random binary sequence generator mounted on top of the data source selection and laser transmitter module. The vertical housing in the background is the Clock generator. 


\section{TRIAL PREPARATION AND MEASUREMENT CAMPAIGN}

\subsection{Thermal Measurements}

One overall program objective was to gain experience designing optical communication systems that can operate successfully in the harsh stratospheric conditions. Thermal modelling and testing were accomplished during the system design to account for temperatures down to $-70^{\circ} \mathrm{C}$. During the design phase, several prototype subsystems such as the pointing assembly were tested in a small thermal chamber to optimize and validate the design. The FELT was tested in a thermal vacuum chamber prior to the balloon flight in order to validate the passive thermal control approach and the system design. Fig. 8 shows a picture of the FELT setup in the thermal vacuum chamber prior to testing.

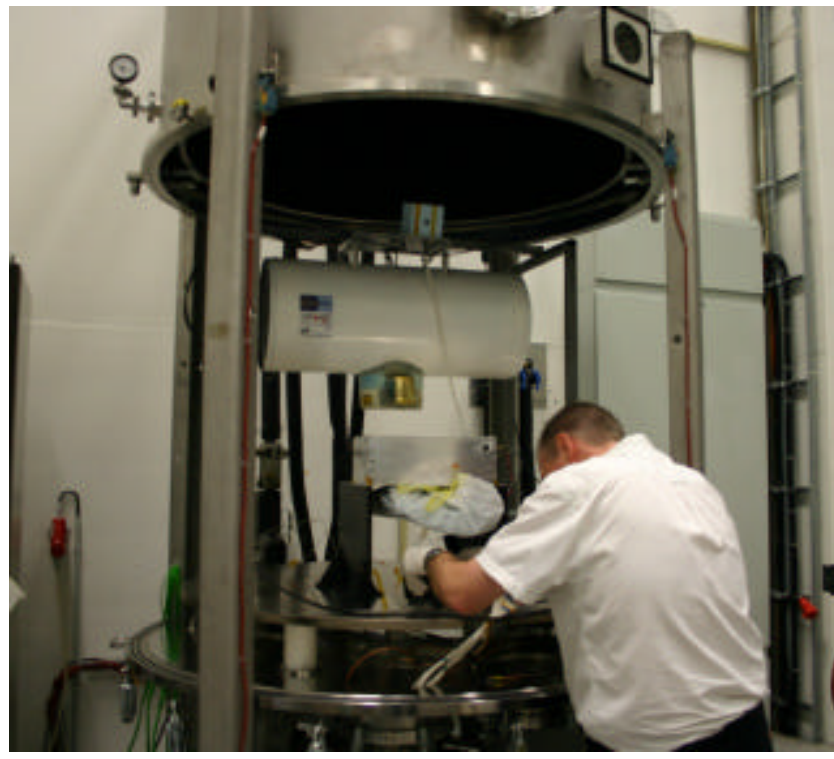

Fig. 8. FELT in thermal vacuum chamber

The FELT performed well during the thermal vacuum test with no anomalous behavior. Fig. 9 shows the temperature measurements from selected thermocouples on the FELT during the thermal vacuum test. The effective minimum temperature of the chamber was about $-65^{\circ} \mathrm{C}$ (thermocouple placed on the inside surface of the chamber). The air temperature inside the chamber was approximately $-45^{\circ} \mathrm{C}$ (thermocouple hanging free in the chamber). However, the air temperature reading from the thermocouple can not be completely relied upon due to the near vacuum conditions (50 millibar). The carbon fiber housing provided good thermal insulation, which can be seen by comparing the temperature readings on the inside and outside surface of the pod; the inside surface was approximately $30^{\circ} \mathrm{C}$ warmer than the outside surface. In addition, the passive thermal control approach was validated. Most of the components mounted on the plate stabilized between $20-30^{\circ} \mathrm{C}$ during testing at a chamber temperature of $-65^{\circ} \mathrm{C}$. 


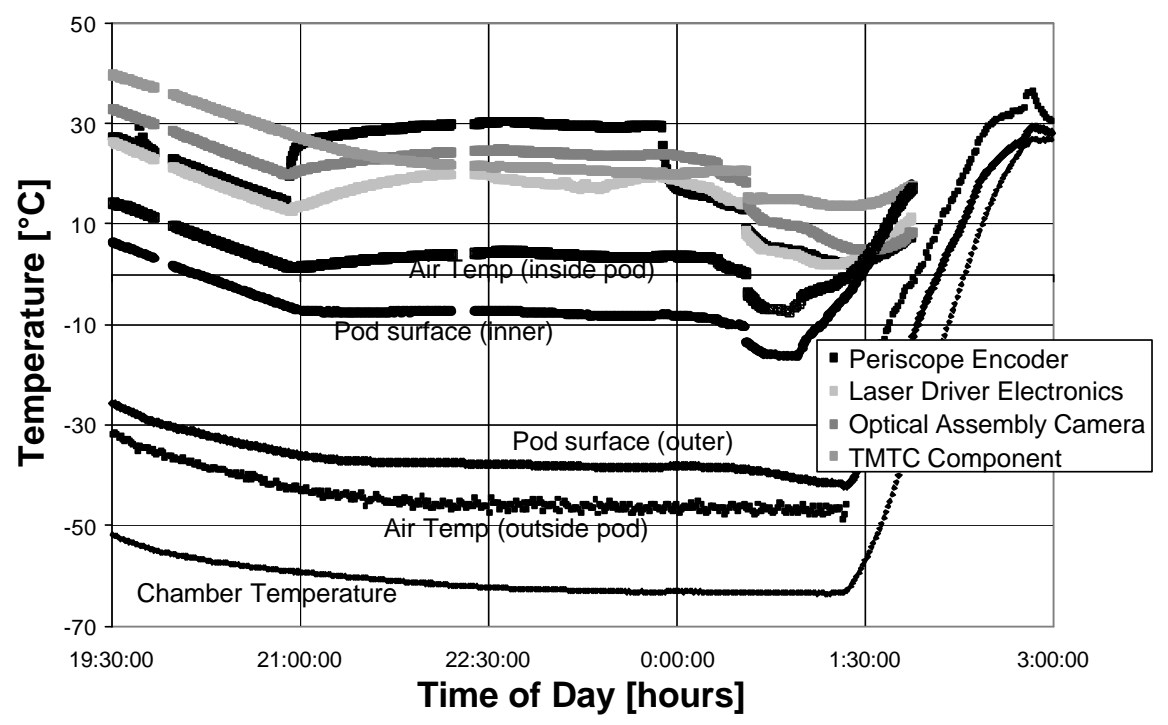

Fig. 9. Selected FELT thermocouple data from the thermal vacuum chamber test

\subsection{Payload Integration}

The measurement campaign took place at the ESRANGE facilities in northern Sweden near Kiruna. Prior to balloon launch, the FELT was integrated into the nacelle of the stratospheric balloon carrier system along with the Millimeterwave CAPANINA payload (Fig. 10). The solar radiation protection cover and retro reflectors can be seen on top of the nacelle. These reflectors were added as a contingency plan in case the FELT malfunctioned. They could have reflected the ground station beacon lasers and minimal TOGS experiments/measurements could have been performed.
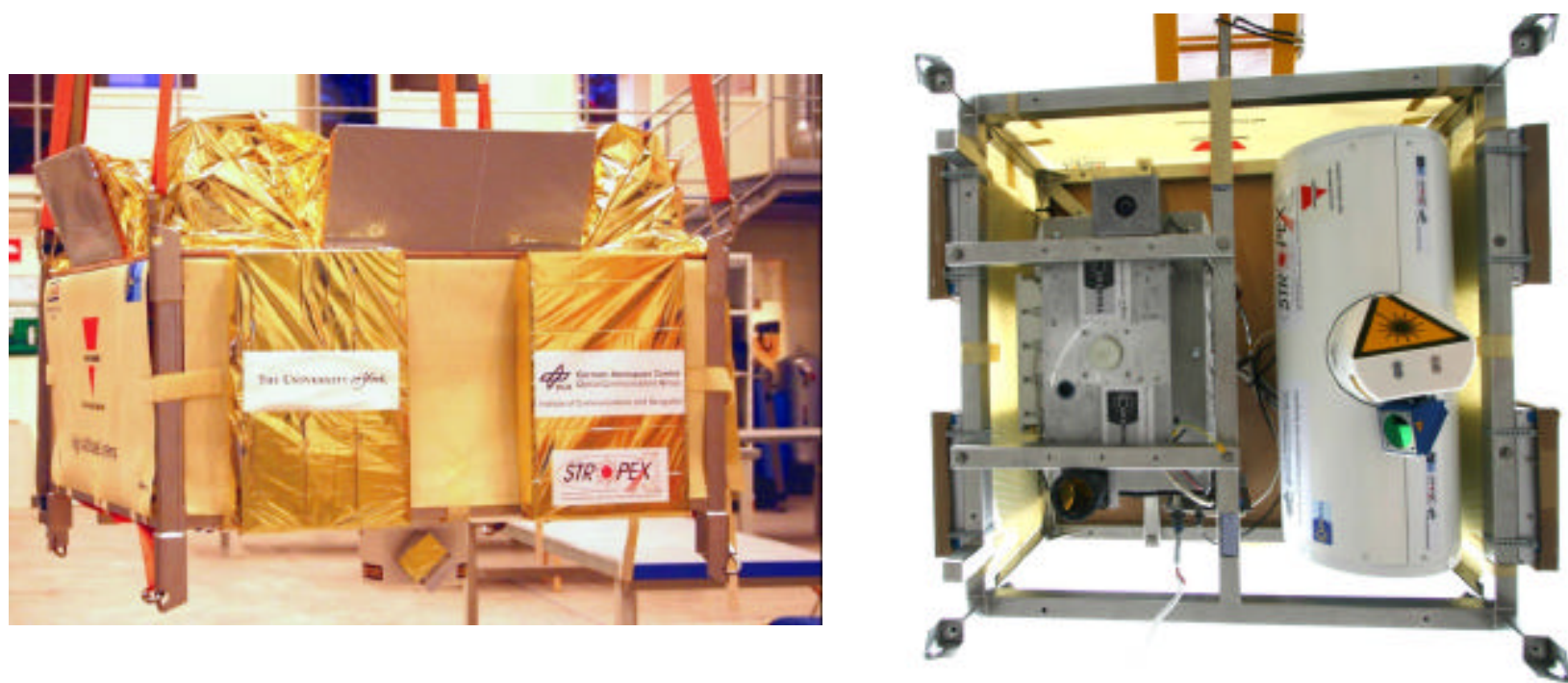

Fig. 10. Integrated CAPANINA payload. Millimeter-wave and optical payload (FELT) integrated into the nacelle. Front view (left) in the integration room and bottom view on the launch vehicle (right). Retro reflectors are attached at the top of the payload which is also covered with solar radiation protection foil.

\subsection{Launch and Operations}

A $12000 \mathrm{~m}^{3}$ piloted balloon carrying the FELT was launched at 3:54 a.m. on August 30, 2005 and flew for approximately 8.5 hours before parachuting to the ground. During the mission, data transmission tests and atmospheric 
measurements were carried out. Fig. 11 shows pictures of the balloon at launch. The launch used a dynamic-release launch technique. The payload was carried by a launch crane which followed the balloon and released the payload at the exact vertical position of the flight train. The long flight train can be seen hanging from the bottom of the balloon. The nacelle, which housed the FELT, was located at the bottom of the flight train.
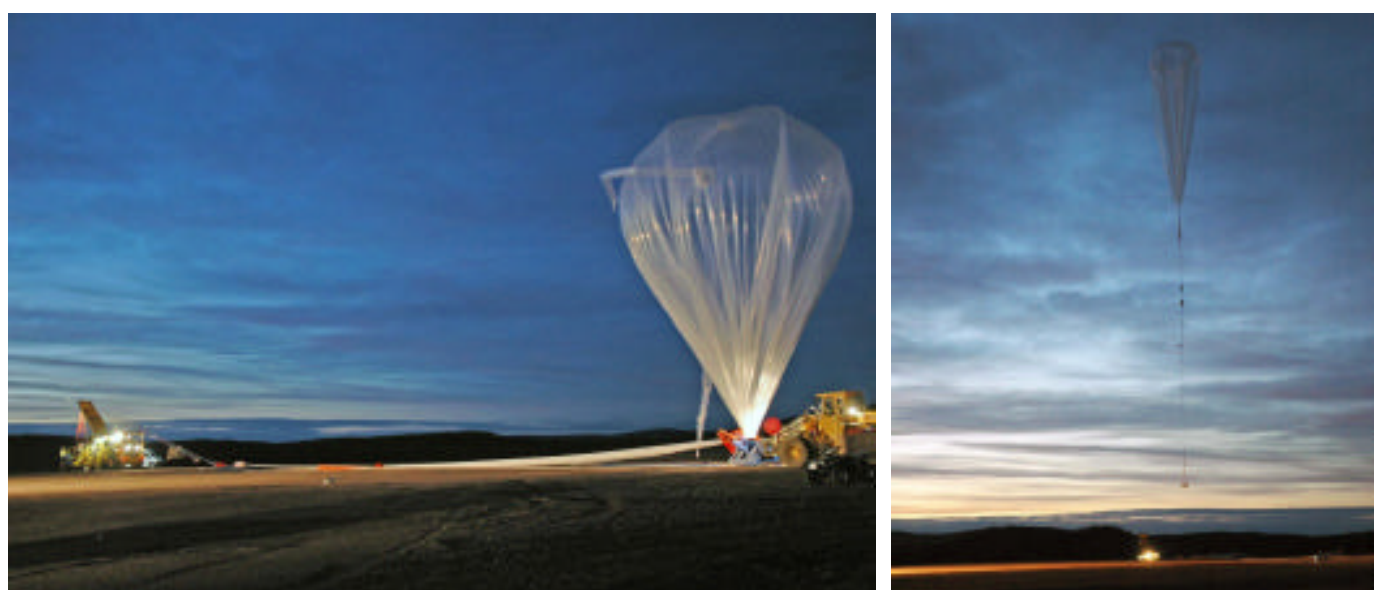

Fig. 11. Picture of balloon before launch with payload at the launch vehicle in the background (left) and flight train at launch (right). The nacelle is located at the bottom of the flight train.

Fig. 12 shows a map of the region surrounding ESRANGE as well as a ground track of the balloon over the duration of the mission. Winds caused the balloon to drift away from the TOGS until the balloon pilot was able to maneuver the balloon into a stratospheric layer with a wind direction that brought the balloon back toward the launch site.

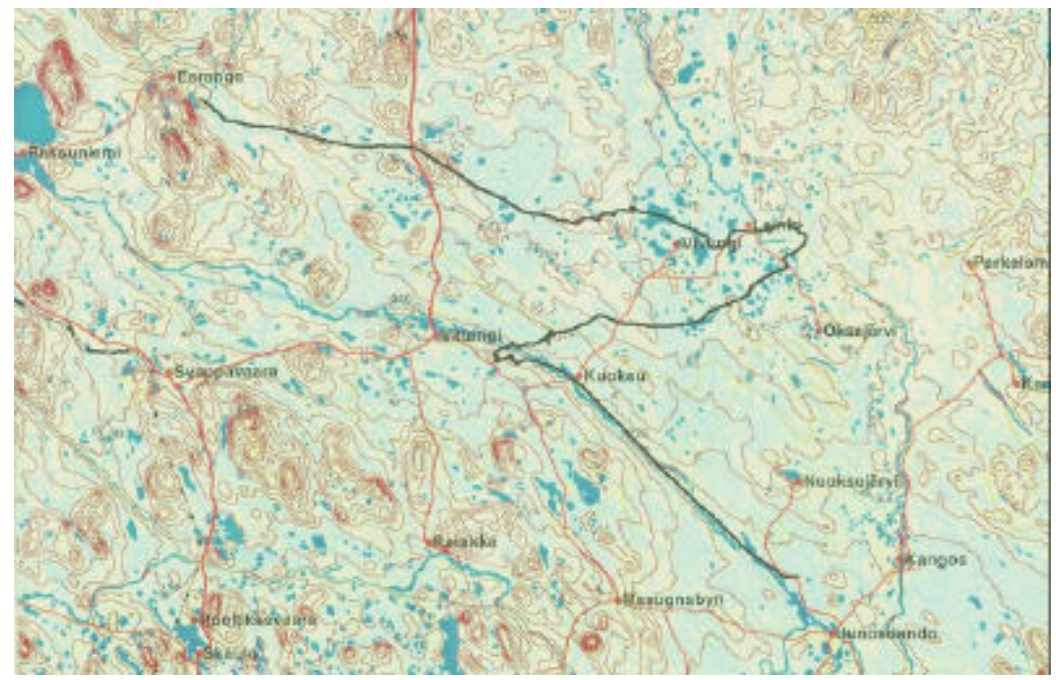

Fig. 12. Map of the region surrounding ESRANGE and a ground track of the balloon.

The maximum range during the mission was $64.1 \mathrm{~km}$ and occurred at 07:03 a.m. local time. Although the communication system was designed for a targeted range of $60 \mathrm{~km}$, optical transmission tests were still successful at the maximum range of $64 \mathrm{~km}$. At this maximum range, the elevation angle from the TOGS to the FELT was about 21 degrees. Fig. 13 shows the range from the TOGS to the balloon during the mission. Fig. 14 shows a plot of the balloon altitude. The balloon pilot was able to keep the balloon near the $22 \mathrm{~km}$ target altitude for the duration of the mission. 


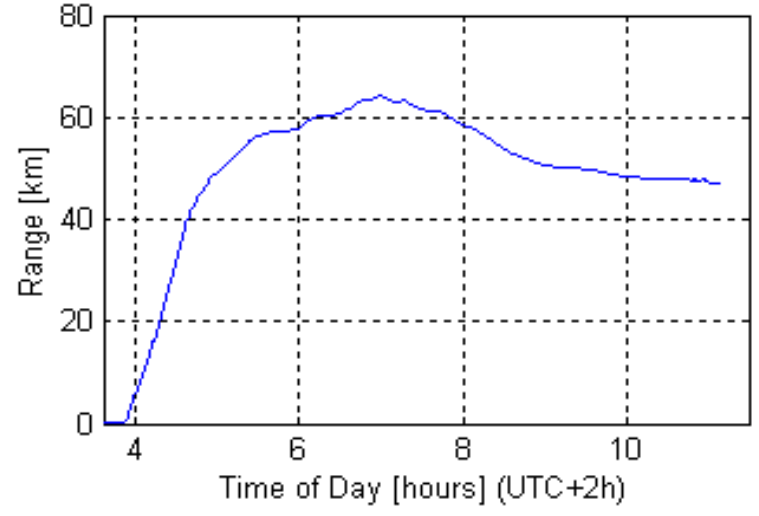

Fig. 13. Plots showing the range from the TOGS to the balloon.

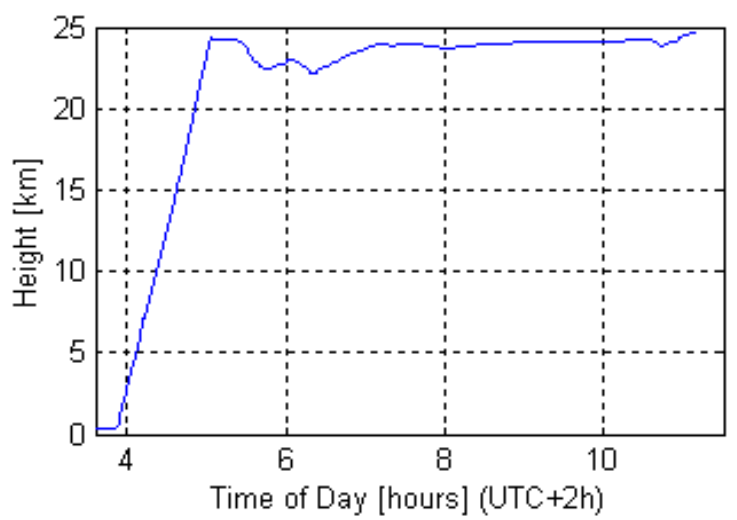

Fig. 14. Plot showing the balloon height during the mission.

\subsection{Acquisition and Tracking}

Immediately after takeoff, the TOGS tracked the balloon via open loop GPS-tracking [6]. The GPS position of the balloon was downlinked via the RS 422 telemetry service link. Seventy minutes after launch, the balloon reached the targeted altitude at a horizontal distance of $48.4 \mathrm{~km}$ from the ground station (Fig. 13 and Fig. 14). At this point the TOGS laser beacons were switched on. Based on the GPS positions of the balloon and the TOGS, the TOGS calculated the initial elevation pointing angle for the FELT and sent this data to the FELT via the telemetry link. Using this elevation angle and the nacelle rotation speed, the FELT PAT computer directed the pointing assembly to scan a circular pattern at a predetermined rotation rate. A scan was necessary for acquisition because the FELT had no way to determine absolute heading angle. The scan rate was chosen in order to minimize the acquisition time.

Once the FELT began scanning for the ground beacon, the establishment of the optical communications link occurred as follows: When the FELT tracking camera detected any bright objects, the size and circumference of the object was measured and compared to expected ground station spot sizes. With this information, it was possible to distinguish between the ground station beacons and spurious background light (e.g. sun reflections on water or glass). If any object passed this test, the system switched from acquisition to tracking mode. In tracking mode, the two periscope axes were driven by a PID position (or rate) controller. The periscope axes were positioned in order to hold the detected object in the center of the tracking camera image. The tracking camera image size was decreased in the tracking mode. This had the advantage of a high control loop bandwidth. Since the tracking camera and the FELT lasers were co-aligned [5], the FELT lasers could be pointed precisely using this tracking method. When the FELT started tracking the ground beacon successfully, the FELT beacon lasers were turned on as seen in Fig. 15.
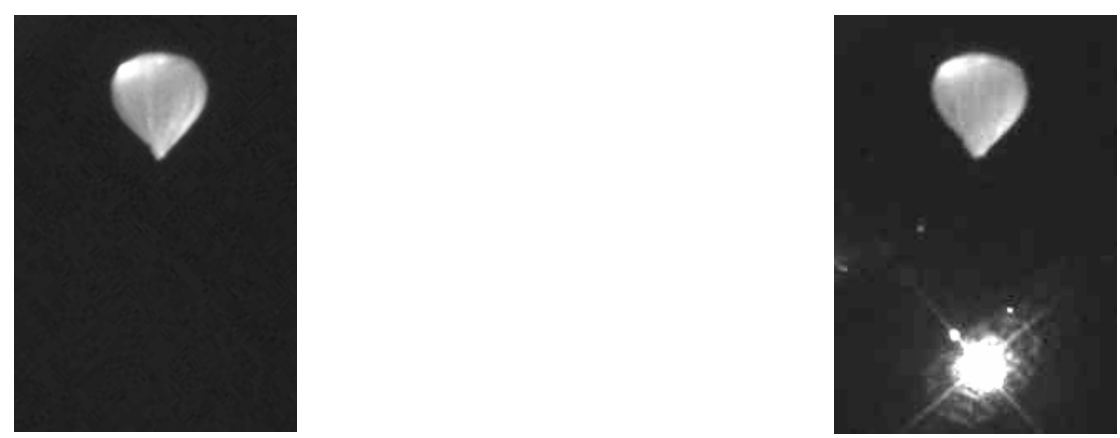

Fig. 15. Near infrared picture from the ground station tracking camera of the stratospheric balloon. Before (left) and after (right) acquisition. The altitude is $23 \mathrm{~km}$ and the distance to the ground station approximately $60 \mathrm{~km}$ 
At this point, the TOGS switched from GPS tracking to closed-loop optical tracking and kept the balloon beacon within the $100 \mu \mathrm{rad}$ field of view (FOV) of the ground station's communication receiver. Finally, the $1550 \mathrm{~nm}$ communication system on the FELT was activated and the data transmission tests were performed.
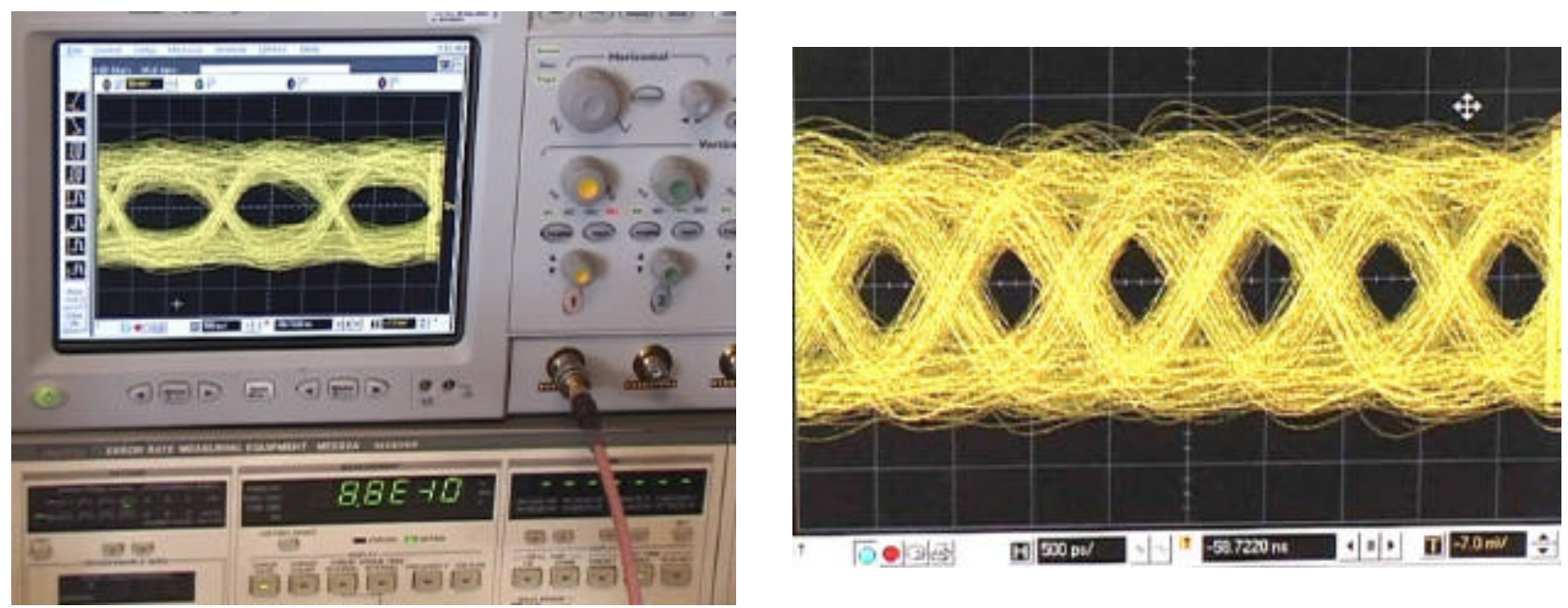

Fig. 16: Received signal PRBS eye patterns. $622 \mathrm{Mbit} / \mathrm{s}$ eye pattern with bit error rate measurement receiver (left picture) and $1.25 \mathrm{Gbit} / \mathrm{s}$ eye pattern (right picture) at approximately $60 \mathrm{~km}$ range.

The high signal to noise ratio enabled a bit error rate (BER) of less than $10^{-9}$ within an unlimited measurement interval at a bitrate of $622 \mathrm{Mbit} / \mathrm{s}$. Frequently, the BER went down to $10^{-10}$ for several minutes. This high quality signal was received up to a maximum distance of $64.15 \mathrm{~km}$ from the ground station. This was the distance when the pilot succeeded in maneuvering the balloon back toward the launch site. The $622 \mathrm{Mbit} / \mathrm{s}$ eye pattern can be seen in the left picture in Fig. 16.

The $1.25 \mathrm{Gbit} / \mathrm{s}$ transmission was also successful. Due to the limited bandwidth of the bit error rate test receiver, no direct BER measurements could be performed at $1.25 \mathrm{Gbit} / \mathrm{s}$. The eye pattern of the received $1.25 \mathrm{Gbit} / \mathrm{s}$ can be seen in the right picture of Fig. 16.

During the flight, clouds periodically disturbed the transmission tests. Several thick black clouds prevented communication totally. Thin white clouds did not significantly effect the transmission. Late in the morning, most of the clouds disappeared giving near clear-sky conditions. During that time, the angular separation between the sun and the balloon was very small, about $5^{\circ}$. Despite an increase of background radiation, which could have affected the intensity modulated signal reception with direct detection (IM/DD), no impairment of link quality was observed. This positive effect was achieved by the narrow filtering $(10 \mathrm{~nm})$ of the incoming $1550 \mathrm{~nm}$ signal and the reduced sun-radiance at this mid-IR wavelength -- a clear advantage of the $1550 \mathrm{~nm}$ technology over NIR wavelengths like $850 \mathrm{~nm}$.

Acquisition of the ground station was very reliable during night and daytime conditions. The maximum acquisition time depended on the scan speed. During all acquisition tests, the maximal time was about 30 seconds at a scan speed of $10 \%$. During the 8 hour trial, $75 \%$ of the time, the FELT tracking deviation was less than $142 \mu \mathrm{rad}(0.0081 \mathrm{deg})$ which equals one pixel of the PAT camera. During the other $25 \%$ of the time, acquisition tests were being performed, clouds prevented the tracking, or stratospheric wind shear caused relatively high pendulousness of the nacelle. Compared to the high beam divergence of $1 \mathrm{mrad}$, the margin of the tracking system was very high and did not lead to a remarkable decrease of communication system performance.

Coincident with the data transmission tests, atmospheric measurements were also carried out. The measurement instruments in the ground station took advantage of the beacon lasers, which allowed the use of off the shelf silicon detector technology. A detailed description of the transportable optical ground station, the turbulence instruments, and the measurement results can be found in [7]. 
At the end of the successful mission, the flight termination system was armed and the nacelle was cut away from the balloon (12:18 pm) and parachuted to the ground. To protect the payloads from ground impact, the nacelle was capsized and crash pads were installed on the top side of the nacelle. After 37 minutes of decent from the stratosphere, the payload touched down at 12:55 pm and recovered without damage. The landing site can be seen in Fig. 17.

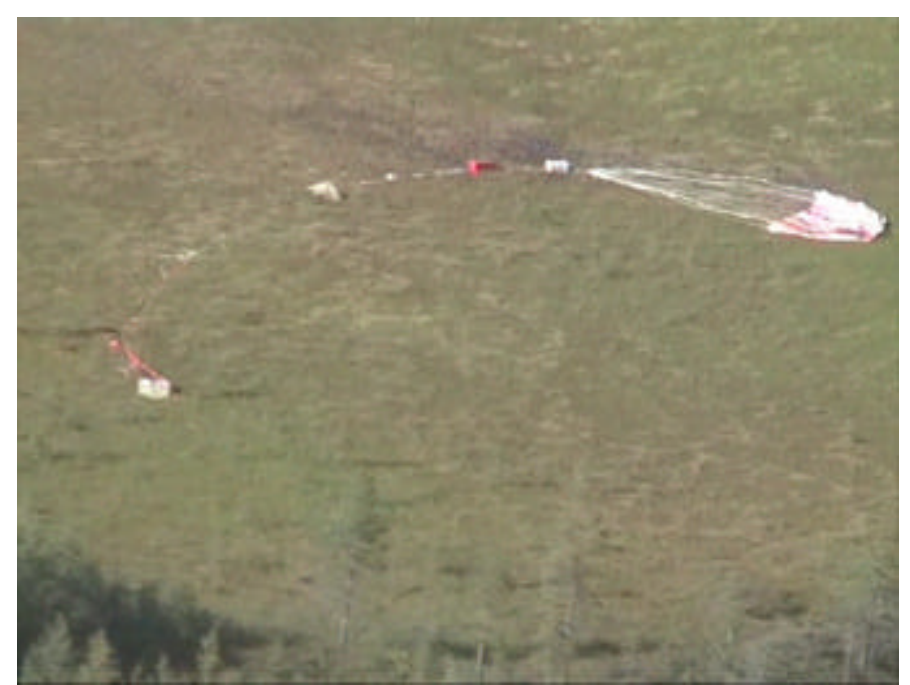

Fig. 17. Payload after touchdown

\section{CONCLUSION AND OUTLOOK}

CAPANINA STROPEX, the first known stratospheric optical high bitrate downlink, was a success. A nearly error free signal was received by the optical ground station at a maximum bitrate of $1.25 \mathrm{Gbit} / \mathrm{s}$ at a link distance of $64 \mathrm{~km}$. The FELT demonstrated good pointing accuracy and tracking performance in the harsh stratospheric conditions. The FELT tracking camera's $4^{\circ}$ field of view could be decreased for a future system, leading to better tracking resolution. With the corresponding decreased divergence angle of the communication laser, the link margin/distance could be increased dramatically with the same transmission power. Future system can easily rely on passive thermal control with nominal thermal insulation.

Knowledge gained in this program will be applied to future optical communication terminal designs. Additionally, with gathered turbulence data, the numerical turbulence simulation tool PiLab will be optimized [10], [11]. Optical system parameters such as aperture size can be defined appropriately according to turbulence conditions and modulation scheme. Furthermore, turbulence mitigation techniques can be designed accordingly [12].

\section{ACKNOWLEDGEMENT}

This project was supported by the European Commission; contract number FP6-IST-2003-506745.

\section{REFERENCES}

1. CAPANINA consortium, http://www.capanina.org

2. D. Grace, M. Mohorcic, J. Horwath, M. B. Pallavicini, M. Fitch, "Integrating Users into the Wider Broadband Network via High Altitude Platforms," IEEE - Int. J. on Wireless Information Networks - Special Issue on Communications via HAPs: Technologies and Trials, Q4-2005.

3. D. Giggenbach, R. Purvinskis, M. Werner, M. Holzbock, "Stratospheric Optical Inter-Platform Links for High Altitude Platforms," AIAA - Proceedings of the $20^{\text {th }}$ ICSSC, Montreal, May 2002. 
4. Oodo, M.; Tsuji, H.; Miura, R.; Maruyama, M.; Suzuki, M.; Nishi, Y.; Sasamoto, H, 'Experiments on IMT-2000 Using Unmanned Solar Powered Aircraft at an Altitude of $20 \mathrm{~km}$ ", Vehicular Technology, IEEE Transactions on Volume 54, Issue 4, July 2005 Page(s): 1278 - 1294

5. J. Horwath, D. Grace, D. Giggenbach, M. Knapek, N. Perlot, "Optical communication from HAPs - Overview of the Stratospheric Optical Payload Experiment (STROPEX)", AIAA - Proc. of the 22 $2^{\text {nd }}$ Int. Com. Satellite Systems Conf. (ICSSC), May 2004.

6. D. Giggenbach, J. Horwath "Optical Free-Space Communications Downlinks from Stratospheric Platforms Overview on STROPEX, the Optical Communications Experiment of CAPANINA," Proceedings of 2005 IST Mobile Summit, Dresden, 2005.

7. Markus Knapek, Joachim Horwath, Nicolas Perlot and Brandon Wilkerson, "The DLR Ground Station in the Optical Payload Experiment (STROPEX) - Results of the Atmospheric Measurement Instruments", Proceedings of the SPIE 2006, Vol. 6304

8. Transportable optical ground station in a stratospheric balloon trial and turbulence measurements" Proceedings of the $3^{\text {rd }}$ Advanced Satellite Mobile Systems Conference (ASMS 2006), Herrsching, May 2006.

9. M. Knapek, J. Horwath, et al., "Transportable Optical Ground Station for Free-Space Laser Communications," Proceedings of the $3^{\text {rd }}$ Advanced Satellite Mobile Systems Conference (ASMS 2006), Herrsching, May 2006.

10. J. Horwath, N. Perlot, D. Giggenbach, R. Jüngling, "Numerical simulations of beam propagation through optical turbulence for high-altitude platform crosslinks," Proceedings of the SPIE 2004, Vol. 5338B.

11. J. Horwath, N. Perlot, "Determination of statistical field parameters using numerical simulations of beam propagation through optical turbulence," Proceedings of SPIE 2004, Vol. 5338.

12. D. Giggenbach, et al., "Measurements at a $61 \mathrm{~km}$ Near-Ground Optical Transmission Channel," Free Space Laser Communication Technologies XIV, Proc. of SPIE 2002, Vol. 4635. 